\title{
A ONE-DIMENSIONAL SPOT WELDING MODEL
}

\author{
K. T. ANDREWS, L. GUESSOUS, S. NASSAR, S. V. PUTTA, AND M. SHILLOR \\ Received 3 July 2006; Revised 3 November 2006; Accepted 22 November 2006
}

A one-dimensional model is proposed for the simulations of resistance spot welding, which is a common industrial method used to join metallic plates by electrical heating. The model consists of the Stefan problem, in enthalpy form, coupled with the equation of charge conservation for the electrical potential. The temperature dependence of the density, thermal conductivity, specific heat, and electrical conductivity are taken into account, since the process generally involves a large temperature range, on the order of $1000 \mathrm{~K}$. The model is general enough to allow for the welding of plates of different thicknesses or dissimilar materials and to account for variations in the Joule heating through the material thickness due to the dependence of electrical resistivity on the temperature. A novel feature in the model is the inclusion of the effects of interface resistance between the plates which is also assumed to be temperature dependent. In addition to constructing the model, a finite difference scheme for its numerical approximations is described, and representative computer simulations are depicted. These describe welding processes involving different interface resistances, different thicknesses, different materials, and different voltage forms. The differences in the process due to AC or DC currents are depicted as well.

Copyright (c) 2006 K. T. Andrews et al. This is an open access article distributed under the Creative Commons Attribution License, which permits unrestricted use, distribution, and reproduction in any medium, provided the original work is properly cited.

\section{Introduction}

The process of resistance spot welding (RSW) of metal sheets is commonly used in many industrial settings. For example, it is often used to bind body panels in the automotive industry, and the mechanical and structural integrity of cars depends on the quality of the welds. In this work, we construct and numerically simulate a one-dimensional model for the process. The model is derived from first principles, and, in view of the large temperature range typically involved in the process, the temperature dependence of the electrical conductivity, the density, the specific heat, and thermal conductivity are all taken into

Hindawi Publishing Corporation

Journal of Applied Mathematics

Volume 2006, Article ID 17936, Pages 1-24

DOI 10.1155/JAM/2006/17936 
account. Our aim is to gain a deeper understanding of the processes involved and to provide reasonable quantitative predictions of the process evolution.

In a typical spot welding process, an electrical current is passed through two adjacent metal plates which induces melting of the plates' material. When the electrical power input is shut off, the plates cool down and the molten material solidifies into a nugget, forming a material bond between the two plates. In industry, the process is performed by robot welders with preset "time schedules," that is, the time intervals, usually measured in cycles, for positioning the electrodes in place, passing the current and cooling the plates. It is often the case that these schedules are based simply on trial and error together with experimental verification. The latter is expensive and time consuming since it requires the dismantling of a statistical sample of the plates in order to inspect the strength and form of the nuggets. Clearly, a reliable scientific model can reduce considerably the number of experimental verifications, and cut costs and effort.

Heat conduction with phase change is the main physical mechanism behind RSW, which involves melting and solidification of the workpieces. One of the main difficulties associated with the solution of this type of problem is the appearance of a free boundary where the material changes phase. This boundary appears and expands as the material melts and contracts and disappears as it solidifies. The numerical methods used for this type of problems have generally been categorized as either being front-tracking methods or enthalpy methods $[3,6]$. In the case of a front tracking method, the Stefan condition must be satisfied on the free boundary, while the heat conduction equation is solved on each side of the boundary [14]. This requires accurate knowledge of the location of the boundary. In the case of the enthalpy formulation, explicit knowledge of the boundary location is not required and can be determined after the fact. Moreover, when there are internal heat sources, the front tracking method is likely to generate superheating in the solid while the enthalpy method would generate a so-called "mushy region" $[12,17]$.

A "mushy region" is a two-phase solid/liquid mixture region where the temperature is constant and is equal to the melting temperature of the material. Such two-phase regions appear during melting processes which start in the interior of the solid and are associated with the appearance of superheating which is unstable, changing a solid material into a stable solid/liquid mixture. As energy is added, it is absorbed as latent heat and so the proportion of liquid to solid increases in the region and the temperature remains constant at the melting temperature until all of the material has liquified, at which point the temperature can begin to increase again $[4,12,13]$.

Many studies have been devoted to resistance spot welding and commercial finiteelement-based software packages such as ABAQUS ${ }^{\circledR}$ and Sysweld ${ }^{\circledR}$ now have capabilities to simulate certain aspects of phase-change and welding processes. An extensive literature survey of solidification and melting heat transfer can be found in [20]. A review of the welding literature shows many numerical simulations (usually finite-element-based) of RSW processes, only a few of which are referenced here. These include one-dimensional [8], two-dimensional $[2,5,11,16,18]$, and three-dimensional [10] simulations of different aspects of RSW. For instance, Feulvarch et al. [5] developed a numerical model for the welding of sheets of the same thickness and material that incorporates electrothermal and mechanical effects to investigate the effects of process variables on the RSW nugget size. 
Others, such as $[11,16]$, used the commercial finite element software ABAQUS to couple thermal, electrical, and mechanical modules in the simulation of RSW or to compare the effect of single phase AC and multiphase DC on the weld size and energy consumption [18]. By and large, a temperature-based formulation was used to model the process $[2,5,11,16,18]$, and an assumption of symmetry was made (hence not allowing for the modeling of plates of dissimilar thicknesses or materials). In many cases, the current density applied at the electrode or the Joule heating responsible for the melting of the material was assumed to be uniform $[10,11,16]$. Mathematical analysis of related models, which couple melting with current flow, can be found in $[7,9,12,15,17,19]$ and in the references therein.

In this work, we construct a model using the enthalpy formulation to describe the evolution of the melting process and using the charge conservation equation to describe the evolution of the electrical potential. We allow for the temperature dependence of the density, thermal conductivity, specific heat, and electrical conductivity, since the process temperature range is quite large, on the order of $1000 \mathrm{~K}$. The model is general enough to allow for the welding of plates of different thicknesses or different materials and accounts for variations in the Joule heating through the material thickness due to the dependence of electrical resistivity on temperature. A novel feature in the model is the use of interface resistance between the plates which is also assumed to be temperature dependent. We also introduce the nugget function which keeps track of the molten and mushy regions and which, therefore, measures the size of the solidified nugget. Moreover, it can be used to determine when the electrical and thermal resistances between the two plates vanish. This happens when one of the plates starts melting and the gap between the two fills with molten material, thus reducing the interface resistance.

The rest of the paper is structured as follows. In Section 2, we develop the model. A finite difference algorithm is described in Section 3, while results of its implementation can be found in Section 4. Here we consider simulations which involve different resistances, different thicknesses, different materials, and different voltage forms. The paper concludes in Section 5, which also points out directions for future work.

\section{The model}

A schematic setting of the spot welding process is depicted in Figure 2.1 with further details presented in Figure 2.2. Two metal sheets are pressed together, two cooled electrodes are applied on both outer surfaces, and an electrical potential $V_{0}$ is switched on across a transformer. The resulting electrical current generates heat in the plates via the Joule effect. The temperature increases and eventually a molten region appears and grows. At a preset time, the electrical potential is switched off, the heating ceases, and the molten region solidifies into a solid nugget which bonds the plates.

In industry, this operation is performed by robots on a very large scale. The programming, or the so called "welding schedule," consists of the specification of the potential drop and the time of current flow and contact force. In more sophisticated settings, there is continuous monitoring of the process parameters, and some types of control are applied in "real time." Welding times which are too short may lead to weak welding bonds, while times which are too long are expensive, slow down the operation, and may cause 


\section{A one-dimensional spot welding model}

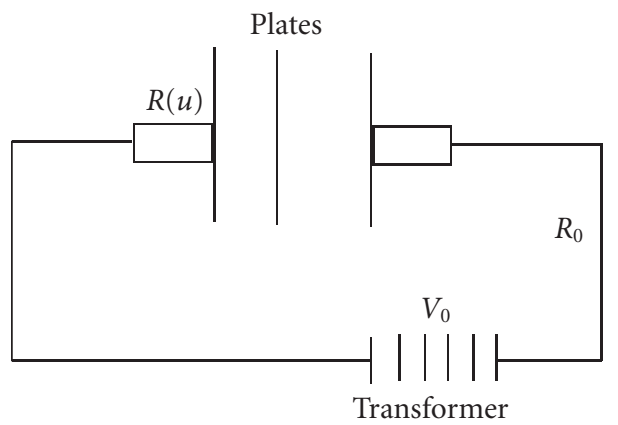

Figure 2.1. The spot welding setting.

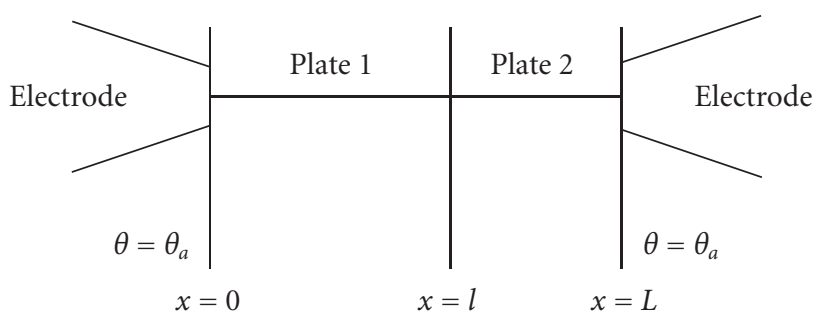

Figure 2.2. Expanded view of the plates and electrodes.

substantial thermal deformations. Even worse, if the molten zone reaches the outer surface of a plate, it may cause flow of liquid metal (also known as expulsion), damaging the electrodes and possibly the robot and causing a safety hazard. Therefore, there is a considerable interest in the optimization of the process "schedule;" the model presented here is a step towards this goal. We assume that the plates are made of different materials and have different thicknesses. However, we describe the case with only two plates; it is easy and straightforward to modify the model to consider the case of three or more plates.

As shown in Figure 2.2, the first plate has thickness $l$ and occupies the interval $0<x<l$; the second plate has thickness $L-l$ and occupies the interval $l<x<L$. We use subscripts $i=1$ and $i=2$ to denote quantities related to the first and second plate, respectively, and everywhere below $i=1,2$. The two electrodes are situated at $x=0$ and $x=L$. For plate $i$, we let $\theta$ denote the temperature, so that $\theta_{i}=\theta_{i}(x, t)$ refers to the temperature distribution at location $x$ and time $t$ in plate $i$. Similarly, the enthalpy per unit mass is $h_{i}=h_{i}(x, t)$. To simplify the notation from this point on we will not use the subscript $i$ unless we wish to describe a specific property of plate $i$. Thus, for example, we will use $h$ to denote the enthalpy of the system, with the understanding that restricted to plate $i$ it is $h_{i}$.

To describe the process of melting, we use not only the thermodynamic enthalpy per unit mass function, $h$, but also the enthalpy per unit volume, $H=\rho h$. Here $\rho=\rho_{i}\left(h_{i}\right)$ denotes the material density. We also let $c=c_{i}\left(\theta_{i}\right)$ denote the temperature dependent 


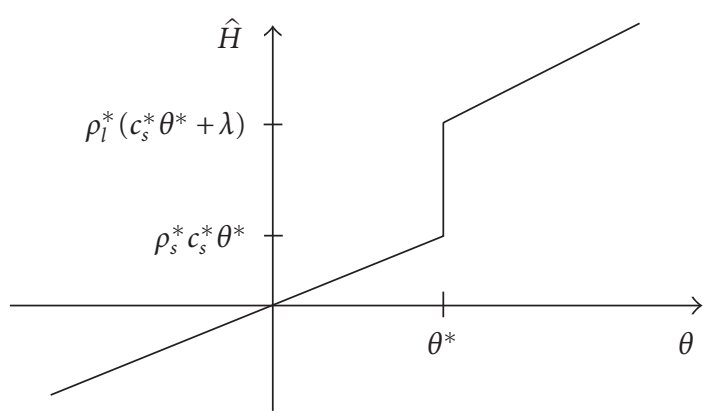

Figure 2.3. The graph of $\hat{H} ; \theta^{*}$ is the melting temperature.

specific heat (we note that $c=d h / d \theta$ ), let $k=k_{i}\left(h_{i}\right)$ denote the thermal conductivity, and let $\sigma_{i}=\sigma_{i}\left(h_{i}\right)$ denote the local electrical conductivity. Finally, we let $\lambda_{i}$ denote the latent heat of melting, and let $\theta_{i}^{*}$ denote the melting temperature of plate $i$, for $i=1,2$. Both of these latter quantities are constant, but we assume that the density, thermal conductivity, and local electrical conductivity depend on the enthalpy, while the specific heat $c$ depends on the temperature, as well as on the phase (solid, liquid, or mixture) of the material. We will describe the dependence on the phases in detail below. We will use subscripts $s$ and $l$ to denote a property value for the solid or liquid (melted) material, respectively.

To describe the relationship between the enthalpy per unit volume $H$, and the temperature $\theta$, we introduce the enthalpy graphs $\hat{H}$ :

$$
\hat{H}_{i}\left(\theta_{i}\right)= \begin{cases}\rho_{i s} c_{i s}\left(\theta_{i}\right) \theta_{i}, & \theta_{i}<\theta_{i}^{*} \\ {\left[\rho_{i s}^{*} c_{i s}^{*} \theta_{i}^{*}, \rho_{i l}^{*}\left(c_{i s}^{*} \theta_{i}^{*}+\lambda_{i}\right)\right],} & \theta_{i}=\theta_{i}^{*} \\ \rho_{i l}\left(c_{i l}\left(\theta_{i}\right) \theta_{i}+\Lambda_{i}\right), & \theta_{i}>\theta_{i}^{*}\end{cases}
$$

Here $c_{i s}^{*}=c_{i s}\left(\theta_{i}^{*}\right)$ and similar definitions are used for the other $*$ variables, while the definition $\Lambda_{i}=\lambda_{i}+\left(c_{i s}^{*}-c_{i l}^{*}\right) \theta_{i}^{*}$ ensures the continuity of the function past the melting point.

The enthalpy per unit volume function $H$ is then a selection out of the enthalpy graph $\hat{H}(\theta)$ given in Figure 2.3. The zone where $\theta_{i}<\theta_{i}^{*}$ represents the solid phase and the zone where $\theta>\theta_{i}^{*}$ the liquid phase in plate $i$. The jump at the melting temperature equals the total latent heat $\rho \lambda$ needed to melt a unit volume of the material.

The zone where the enthalpy per unit volume $H_{i}$ belongs to the interval $\left[\rho_{i s}^{*} c_{i s}^{*} \theta_{i}^{*}\right.$, $\left.\rho_{i l}^{*}\left(c_{i s}^{*} \theta_{i}^{*}+\lambda_{i}\right)\right]$ is the so-called mushy region, in which the temperature is constant and is equal to the melting temperature. This region is of considerable importance in the process; however, what actually happens in it depends on the material microstructure and is not yet well understood. Although the temperature in the mushy region is identically $\theta^{*}$, the enthalpy per unit mass $h$ changes from $c_{s}^{*} \theta^{*}$ to $c_{s}^{*} \theta^{*}+\lambda$ as the material changes from solid to liquid phase. Consequently, we can use this change in enthalpy to measure the change in the ratio of the solid and liquid phases in the region. Indeed, if we denote 
by $\beta_{i}$ the spatially and temporally varying liquid mass fraction in the mushy region

$$
\beta_{i}=\frac{h_{i}-c_{i s}^{*} \theta_{i}^{*}}{\lambda_{i}}=\frac{1}{\lambda_{i}}\left(\frac{H_{i}}{\rho_{i}}-c_{i s}^{*} \theta_{i}^{*}\right),
$$

then when $\beta_{i}=0$ the grain at location $x$ and time $t$ in the $i$ th plate is fully solid (at the temperature $\theta_{i}^{*}$ ); when $\beta_{i}=1$, it is completely molten; and when $0<\beta_{i}<1$ the fraction $\beta_{i}$ of the grain is liquid and $1-\beta_{i}$ is solid. Note that this definition is only valid at locations where $\theta_{i}(x, t)=\theta_{i}^{*}$.

We can now use $\beta$ to take into account the changes which occur in $\sigma, \rho$, and $k$ as the material changes phase. Specifically, we assume that the electrical conductivity $\sigma_{i}$ in the $i$ plate is given by

$$
\sigma_{i}\left(h_{i}\right)= \begin{cases}\sigma_{i s}\left(\theta_{i}\right), & \theta_{i}<\theta_{i}^{*} ; \\ \beta_{i} \sigma_{i l}\left(\theta_{i}^{*}\right)+\left(1-\beta_{i}\right) \sigma_{i s}\left(\theta_{i}^{*}\right), & \theta_{i}=\theta_{i}^{*} ; \\ \sigma_{i l}\left(\theta_{i}\right), & \theta_{i}>\theta_{i}^{*},\end{cases}
$$

where $\sigma_{i s}$ and $\sigma_{i l}$ are the electrical conductivities in the solid and liquid phases of plate $i$. This choice assumes that in the mushy region the electrical conductivity is determined by the fraction of the liquid and solid phases that are present. We make a similar assumption on the thermal conductivity $k$ and the specific heat $c$. In the case of material density, we use

$$
\frac{1}{\rho_{i}}=\frac{1}{\rho_{i s}^{*}}+\beta_{i}\left(\frac{1}{\rho_{i l}^{*}}-\frac{1}{\rho_{i s}^{*}}\right)
$$

when $\theta_{i}=\theta_{i}^{*}$. This ensures that the volume of the solid/liquid mixture is equal to the sum of the liquid and solid volumes.

Clearly, these are the simplest choices for $\sigma, k$, and $c$; other and more complex choices are possible, and, ultimately, the electrical and thermal conductivities in the mushy zone must be determined experimentally. Moreover, since they depend on the microstructure of the material grains, they are likely to be complex and specific to the material being used.

Finally, we let the electrical potential be denoted by $\varphi_{i}=\varphi_{i}(x, t)$, and note that the electrical current through the two plates can be expressed in terms of the electrical conductivity and electrical potential as $I=\sigma(h) \varphi_{x}$, where the subscript $x$ represents the spatial partial derivative, while the Joule heating is given by $J=\sigma(h)\left(\varphi_{x}\right)^{2}$. We can now describe the equations governing the process.

The enthalpy function, in each plate, is a pointwise selection out of the graph

$$
H \in \hat{H}(\theta) \text {. }
$$

The energy equation is

$$
\frac{\partial H}{\partial t}-\frac{\partial}{\partial x}\left(k(H) \frac{\partial \theta}{\partial x}\right)=\sigma(H)\left(\frac{\partial \varphi}{\partial x}\right)^{2}, \quad x \neq l,
$$


and the charge conservation equation is

$$
\frac{\partial}{\partial x}\left(\sigma(H) \frac{\partial \varphi}{\partial x}\right)=0, \quad x \neq l
$$

These latter two equations hold in the interior of the plates $\{x \neq l\}$, but not at the interface $\{x=l\}$.

To complete the model we need to prescribe the initial and boundary conditions, as well as the transmission conditions across the interface $x=l$.

The initial conditions take the form $H_{i}(x, 0)=H_{i 0}(x)$ in plate $i$. However, when the process starts with two solid plates, which is the case in the industrial setting under consideration, we need only prescribe the initial temperature $\theta_{i 0}$, which is usually just the ambient temperature $\theta_{a}$. Then, the enthalpy is found from condition (2.5), that is,

$$
H_{i}(x, 0)=\rho_{i s} c_{i} \theta_{i 0}(x) .
$$

The temperature at the outer surfaces is taken as the ambient temperature $\theta_{1}=\theta_{a}$ at $x=0$ and $\theta_{2}=\theta_{a}$ at $x=L$. It is straightforward to replace this condition with the more realistic heat exchange condition

$$
q=-k_{i} \frac{\partial \theta_{i}}{\partial x}=k_{e x}\left(\theta_{i}-\theta_{i e}\right), \quad x=0, L
$$

where $k_{e x}$ is the heat exchange coefficient, and $\theta_{i e}$ is the electrode temperature. Even more sophisticated conditions may be used, but, for the sake of simplicity, we consider only the Dirichlet condition given above.

Before we continue with the transmission conditions at the interface $x=l$, we introduce the nugget function $\Psi$, which allows us to describe the disappearance of the resistance between the plates at the initiation of melting at $x=l$. But, more importantly, it is designed to keep track of the melting process and to describe the growth of the nugget. We define it in terms of the enthalpy (and not just temperature), since in this manner it provides a more detailed picture of the melting process; thus,

$$
\Psi(x, t)=\max _{0 \leq \tau \leq t} H(x, \tau)
$$

Then the set

$$
N(t)=\left\{x \in[0, l] ; \Psi(x, t) \geq \rho_{1 s}^{*} c_{1 s}^{*} \theta_{1}^{*}\right\} \cup\left\{x \in[l, L] ; \Psi(x, t) \geq \rho_{2 s} c_{2 s}^{*} \theta_{2}^{*}\right\}
$$

describes all the locations at which melting occurred at any time up to and including $t$. This includes all the mushy and molten regions (note: "molten" refers to $\left\{\theta>\theta^{*}\right\}$ ) and therefore gives the maximal extent of the nugget. The part of the nugget that was at some time up to and including $t$ completely molten is given by

$$
N_{\mathrm{cm}}(t)=\left\{x \in[0, l] ; \Psi(x, t) \geq \rho_{1 l}^{*}\left(c_{1 s}^{*} \theta_{1}^{*}+\lambda_{1}\right)\right\} \cup\left\{x \in[l, L] ; \Psi(x, t) \geq \rho_{2 l}^{*}\left(c_{2 s}^{*} \theta_{2}^{*}+\lambda_{2}\right)\right\},
$$


and so the part of the nugget that was never fully molten is given by

$$
N_{\text {mush }}(t)=N(t)-N_{\mathrm{cm}}(t) \text {. }
$$

An important use of the nugget function $\Psi$ occurs in the case when the solidified nugget material has a different microscopic structure than the material of the original plates. In this situation, the nugget region will be clearly distinct from the surrounding region formed by the original two plates.

We turn next to model the resistance of the interface between the plates. This resistance may be affected by the microscopic gaps that occur due to imperfect contact between the two plates and by the insertion of thin coats of materials between the plates. Indeed, a new version of the process was announced in [1] in which a thin layer of a special highly resisting material is inserted between the zinc-coated plates. The purpose of the coating is to speed up the melting process and to use smaller currents. We incorporate this option into the model by including an adjustable interface resistance term.

To conform to industrial practice, we assume that a potential drop $V_{0}$ is applied to the transformer and we allow it to be time dependent, that is, $V_{0}=V_{0}(t)$. Let $R_{0}$ denote the resistance of the cables and electrodes. We denote by $R_{g}$ the interface resistance attributable to microscopic gaps and possible coatings, and we assume that it is a function of the interface temperature. Moreover, we assume that, as melting commences, the gap fills with material and the resistance drops essentially to zero. We assume that it actually does vanish when melting starts at the interface (on the side with the lower melting temperature), and that the process is irreversible, so that once melting takes place, the interface resistance vanishes for all subsequent times. Thus, we assume that $R_{g}$ is a function of the temperature and of the maximum interface temperature

$$
\vartheta=\vartheta(t)=\max _{0 \leq \tau \leq t} \theta(l, \tau)
$$

and that $R_{g}$ vanishes when $\vartheta(t) \geq \vartheta_{\min }^{*}$, where

$$
\vartheta_{\min }^{*}=\min \left\{\theta_{1}^{*}, \theta_{2}^{*}\right\}
$$

Thus,

$$
R_{g}=R_{g}(\theta(l, t), \vartheta(t)), \quad R_{g}=0, \text { if } \vartheta(t) \geq \vartheta_{\text {min }}^{*}
$$

A typical graph of $R_{g}$ versus $\theta(l, t)$ is given in Figure 2.4. The assumption that $R_{g}$ depends on $\theta(l, t)$ is made for the sake of generality, since the interface temperature may fluctuate while $\vartheta$ is constant. However, in a process where the interface temperature rises steadily, this may be redundant. We note that when the two plates are made of the same material, then $\vartheta_{\min }^{*}=\theta^{*}$, and $R_{g}$ vanishes when the interface starts to melt, that is, $\theta(l, t)=\theta^{*}$. 
K. T. Andrews et al. 9

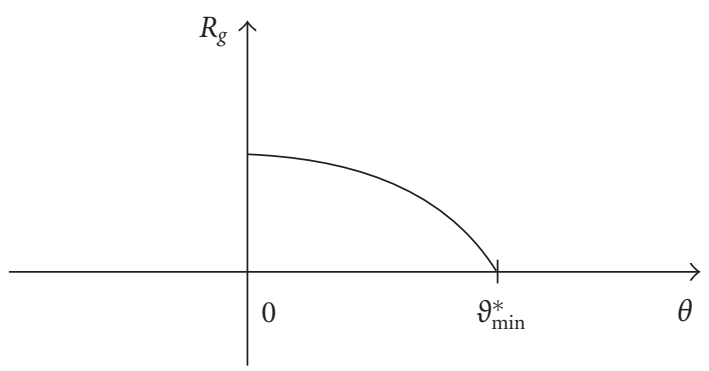

Figure 2.4. The graph of $R_{g}$ versus $\theta(l, t)$.

Recalling that the electrical resistivity is given by $1 / \sigma(H)$, the total resistance per unit area in the transformer loop is $R_{0}+R(H, \theta)$, where

$$
R(t)=R(H, \theta)=\int_{0}^{l} \frac{1}{\sigma_{1}\left(H_{1}(x, t)\right)} d x+\int_{l}^{L} \frac{1}{\sigma_{2}\left(H_{2}(x, t)\right)} d x+R_{g}(\theta(l, t), \vartheta(t))
$$

is the combined resistance of the two plates and the interface. Note that $R(t)$ is time dependent via the dependence of $H, \theta$, and $R_{g}$ on $t$ and, moreover, these two have the dimensions of resistance per unit area $\left(\right.$ say $\Omega / \mathrm{cm}^{2}$ ), as we deal with a one-dimensional setting. For the sake of simplicity, we assume that $R_{0}=$ const.; however, it would cause only minor changes below to assume that it depends on time or even on temperature. We conclude that the current in the loop is $I=V_{0} /\left(R_{0}+R(H, \theta)\right)$, and the potential drop between the electrodes is

$$
V=I R(t)=\frac{V_{0}(t) R(t)}{R_{0}+R(t)}
$$

We are now ready to specify the transmission conditions at $x=l$. We assume that the temperature and the electric current are continuous across the gap, while the electric potential and heat flux jump because of the resistance at the interface. Thus, the following transmission conditions hold:

$$
\begin{gathered}
\theta_{1}(l, t)=\theta_{2}(l, t), \\
\varphi_{1}(l, t)+I R_{g}=\varphi_{2}(l, t), \\
k_{1} \theta_{1 x}(l, t)-I^{2} R_{g}=k_{2} \theta_{2 x}(l, t), \\
\sigma_{1}\left(\theta_{1}(l, t)\right) \varphi_{1 x}(l, t)=\sigma_{2}\left(\theta_{2}(l, t)\right) \varphi_{2 x}(l, t) .
\end{gathered}
$$

The term $I R_{g}$ in (2.20) is the potential drop due to interface resistance, and $I^{2} R_{g}$ in (2.21) is the corresponding interface Joule heating term. Both terms vanish upon the initiation of melting at the interface. 
We summarize the mathematical problem as follows.

Find a triple $\{H, \theta, \varphi\}$ such that (2.5)-(2.7), (2.19)-(2.22) hold, together with the initial conditions

$$
H_{1}(x, 0)=H_{10}(x), \quad 0 \leq x \leq l, \quad H_{2}(x, 0)=H_{20}(x), \quad l \leq x \leq L,
$$

and the boundary conditions

$$
\begin{gathered}
\theta_{1}(0, t)=\theta_{2}(L, t)=\theta_{a} \\
\varphi_{1}(0, t)=0 \\
\varphi_{2}(L, t)=\frac{V_{0}(t) R(t)}{R_{0}+R(t)}
\end{gathered}
$$

for $0 \leq t \leq T$, where $T$ is the final time.

We now show that the one-dimensional model (2.5)-(2.26) decouples, resulting in a problem for the temperature and enthalpy only. Once these are found, the electrical potential can be obtained in closed form by integration. To achieve the decoupling, we integrate (2.7), for $i=1$, from 0 to $x$ and obtain that

$$
\left(\sigma_{1}\left(h_{1}\right) \varphi_{1 x}\right)(x, t)=\left(\sigma_{1}\left(h_{1}\right) \varphi_{1 x}\right)(0, t)=I_{1}(t)
$$

that is, the current is independent of $x$. Similarly, we integrate, for $i=2$, from $l$ to $x$ and find that

$$
\left(\sigma_{2}\left(h_{2}\right) \varphi_{2 x}\right)(x, t)=\left(\sigma_{2}\left(h_{2}\right) \varphi_{2 x}\right)(l, t)=I_{2}(t)
$$

Then, it follows from (2.22) that $I_{1}(t)=I_{2}(t)=I(t)$. Furthermore, another integration over $0 \leq x \leq L$ and simple manipulations using the transmission conditions (2.21) and (2.22) yield $I(t)=V_{0} /\left(R_{0}+R(t)\right)$, where the total resistance $R(t)$ is given in (2.17). The Joule heating terms in (2.6) then become

$$
\sigma_{i}\left(H_{i}\right)\left(\varphi_{i x}\right)^{2}=\frac{I^{2}(t)}{\sigma_{i}\left(H_{i}\right)}, \quad i=1,2 .
$$

For notational convenience, let

$$
F(\sigma(H), \theta)=\frac{V_{0}^{2}(t)}{\sigma(H(x, t))\left(R_{0}+R(t)\right)^{2}} .
$$

The decoupled problem may now be stated. 
Find a pair $\{H, \theta\}$ such that

$$
\begin{gathered}
H \in \hat{H}(\theta), \quad x \neq l, \\
\frac{\partial H}{\partial t}-\frac{\partial}{\partial x}\left(k(H) \frac{\partial \theta}{\partial x}\right)=F(\sigma(H), \theta), \quad x \neq l, \\
I(t)=\frac{V_{0}(t)}{R_{0}+R(t)}, \\
k_{1} \theta_{x}(l-, t)-I^{2} R_{g}=k_{2} \theta_{x}(l+, t), \quad 0 \leq t \leq T, \\
h(x, 0)=h_{0}(x), \quad 0 \leq x \leq L, \\
\theta_{1}(0, t)=\theta_{2}(L, t)=\theta_{a}, \quad 0 \leq t \leq T .
\end{gathered}
$$

Here $F$ is given in (2.30) and $R$ in (2.17).

We note that the decoupled problem is nonlinear and nonlocal, since $F$ depends on the integral of the solution.

Once a solution $\{H, \theta\}$ has been found, the electrical potential $\varphi$ is given by

$$
\begin{gathered}
\varphi_{1}(x, t)=I(t) \int_{0}^{x} \frac{1}{\sigma_{1}\left(\theta_{1}(\xi, t)\right)} d \xi, \quad 0 \leq x \leq l, \\
\varphi_{2}(x, t)=I(t)\left(R_{g}+\int_{0}^{l} \frac{1}{\sigma_{1}\left(\theta_{1}(\xi, t)\right)} d \xi+\int_{l}^{x} \frac{1}{\sigma_{2}\left(\theta_{2}(\xi, t)\right)} d \xi\right), \quad l \leq x \leq L .
\end{gathered}
$$

A formal existence proof for solutions to problem (2.31)-(2.36) is at present an unresolved issue. The nonlinearities in the problem and its lack of smoothness make it a very hard problem for mathematical analysis.

\section{Numerical discretization}

Equations (2.32)-(2.36) were discretized using finite difference approximations. Secondorder central differencing was used for second derivatives, while forward and backward differencing were used to evaluate first derivatives in the first and second plate, respectively. An explicit forward Euler scheme was used for the temporal discretization.

Let $n_{x}$ represent the number of grid points in plate 1 and let $\left(N_{x}-n_{x}\right)$ be the number in plate 2. A uniform grid spacing was assumed for each plate, that is,

$$
\Delta x_{1}=\frac{l}{n_{x}-1}, \quad \Delta x_{2}=\frac{L-l}{N_{x}-n_{x}} .
$$

Let subscript $j$ denote the value of a function at a grid point $x_{j}$, let $\Delta t$ denote the time step size and let superscript $n$ denote the discretized time level. Note that $j=1, j=n_{x}$, and $j=N_{x}$, respectively, correspond to the locations $x=0, x=l$ (interface), and $x=L$. Equation (2.32) was discretized at the interior points, excluding the interface between the two plates, as follows:

$$
\frac{H_{j}^{n+1}-H_{j}^{n}}{\Delta t}=\frac{1}{\Delta x_{i}^{2}}\left(\left(k_{j}^{n}+k_{j+1}^{n}\right)\left(\theta_{j+1}^{n}-\theta_{j}^{n}\right)-\left(k_{j-1}^{n}+k_{j}^{n}\right)\left(\theta_{j}^{n}-\theta_{j-1}^{n}\right)\right)+F_{j}^{n}
$$

for $2 \leq j \leq n_{x}-1$ (in plate $1, i=1$ ) and for $n_{x}+1 \leq j \leq N_{x}-1$ (in plate $2, i=2$ ). 
The Joule heating terms were evaluated as

$$
F_{j}^{n}=\frac{\left(V_{0}^{n}\right)^{2}}{\sigma_{j}^{n}\left(R_{0}+R^{n}\right)},
$$

where a trapezoidal numerical integration was used to evaluate (2.17), that is,

$$
R^{n}=\sum_{j=1}^{n_{x}-1} \frac{\Delta x_{1}}{2}\left(\frac{1}{\sigma_{j}^{n}}+\frac{1}{\sigma_{j+1}^{n}}\right)+\sum_{j=n_{x}}^{N_{x}-1} \frac{\Delta x_{2}}{2}\left(\frac{1}{\sigma_{j}^{n}}+\frac{1}{\sigma_{j+1}^{n}}\right)+R_{g}^{n} .
$$

The temperatures of the outside surfaces were set at all times to the ambient value, that is, $\theta_{1}^{n+1}=\theta_{N_{x}}^{n+1}=\theta_{a}$. The interior grid point temperatures were evaluated by comparing the $H_{j}^{n+1}$ values to the melting temperature solid and liquid enthalpy per unit volume values, $H_{i s}^{*}=\rho_{i s}^{*} c_{i s}^{*} \theta_{i}^{*}$ and $H_{i l}^{*}=\rho_{i l}^{*}\left(c_{i s}^{*} \theta_{i}^{*}+\lambda_{i}\right)$, respectively, using (2.1).

At the interface $j=n_{x}$, the transmission condition was discretized as shown below in (3.5) to obtain the interface temperature, $\theta_{n_{x}}$, at time level $n+1$ :

$$
k_{n_{x}-1}^{n+1} \frac{\theta_{n_{x}}^{n+1}-\theta_{n_{x}-1}^{n+1}}{\Delta x_{1}}=I^{2} R_{g}+k_{n_{x}+1}^{n+1} \frac{\theta_{n_{x}+1}^{n+1}-\theta_{n_{x}}^{n+1}}{\Delta x_{2}} .
$$

The interface resistance $R_{g}^{n}$ was, for the sake of simplicity, taken to vary linearly with the interface temperature, starting from an initial value $R_{g 0}$ and ending at zero according to (2.16). Once the value of $R_{g}$ reaches zero, it is not allowed to return back to a nonzero value, even when the voltage is shut off and solidification occurs.

In locations where the temperature is equal to the melting temperature, the liquid mass fraction, $\beta_{i}$, is evaluated. This fraction is needed to evaluate the various material properties in the mushy zone. As can be seen from (2.2) and (2.4), finding $\beta_{i}$ requires knowing $\rho_{i}$, which in turn depends on $\beta_{i}$. This problem is circumvented by substituting (2.4) into (2.2) and solving for $\beta_{i}$,

$$
\beta_{i}=\frac{H_{i} / \rho_{i s}^{*}-c_{i s}^{*} \theta_{i}^{*}}{\lambda_{i}-H_{i}\left(1 / \rho_{i l}^{*}-1 / \rho_{i s}^{*}\right)} .
$$

In the nonmushy zones, the solid and liquid material property values are determined at each time level and at every grid point using linear interpolation of property values with respect to reference temperatures in the solid and liquid phases, as well as at the melting temperature.

\section{Numerical experiments}

In this section, we discuss numerical experiments that were conducted to test the model implementation and that depict the behavior of the resulting numerical solutions.

Unless otherwise specified, the plate material used was aluminum, which has a melting temperature of $933.2 \mathrm{~K}$ and a latent heat of melting (or fusion) of $3.97 \times 10^{5} \mathrm{~J} / \mathrm{kg}$. Aluminum was selected for this study in part because of the difficulties encountered when trying to weld aluminum sheets. The ambient temperature was assumed to be $300 \mathrm{~K}$. In 

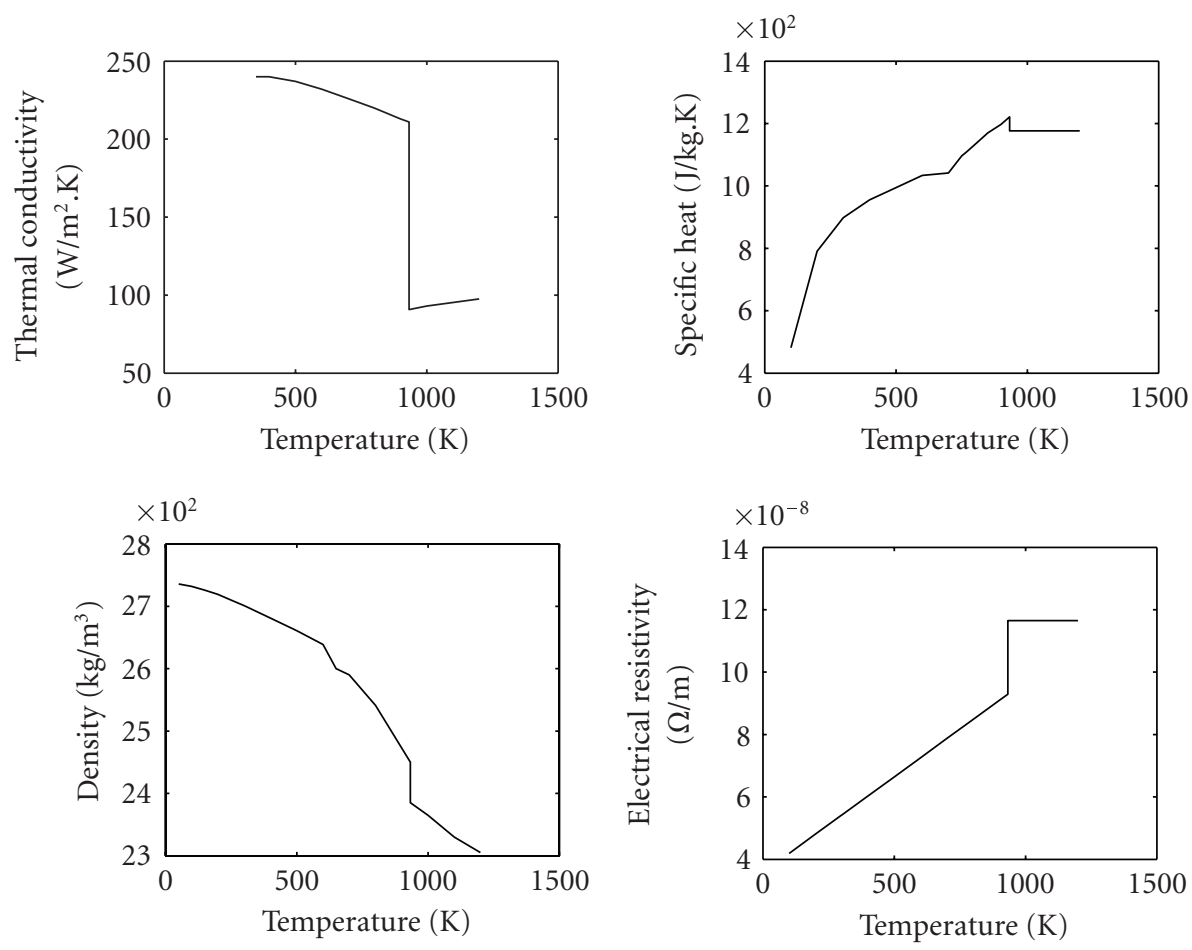

Figure 4.1. Temperature dependent properties for Aluminum. The melting temperature is $933.2 \mathrm{~K}$.

all cases, a spatial and temporal grid independence study was conducted to ensure that the results were grid independent. For a plate thickness of $6 \mathrm{~mm}, 31$ grid points were used, that is, $n_{x}=31$ and $N_{x}=61$. In most cases, the voltage was shut off when the interface temperature reached $1050 \mathrm{~K}$, the two plates were allowed to cool back down and the nugget to solidify, thus binding the two plates. The resistance $R_{0}$ of the cables and electrodes was taken as $10^{-7} \Omega / \mathrm{m}^{2}$, and the voltage was set at $V_{0}=70 \mathrm{~V}$.

Before proceeding to a discussion of the various test cases, it seems worthwhile to describe the effect of temperature on the aluminum material properties used in this study. Figure 4.1 shows the variation of thermal conductivity, specific heat, density, and electrical resistivity with temperature. We note that all of the properties exhibit a sudden jump in value at the melting temperature. The values on either side of the drop represent the solid and liquid properties at the melting temperature, that is, $k_{l}^{*}$ and $k_{s}^{*}$ in the case of thermal conductivity. All of the properties, except for electrical resistivity (the inverse of electrical conductivity), experience a drop in value as the aluminum melts. It must be noted that locating solid and molten property values in the engineering literature was quite difficult. In the cases of specific heat and electrical resistivity, only one property value could be found in the liquid phase, so these two properties were assumed to be constant for temperatures above the melting temperature. 


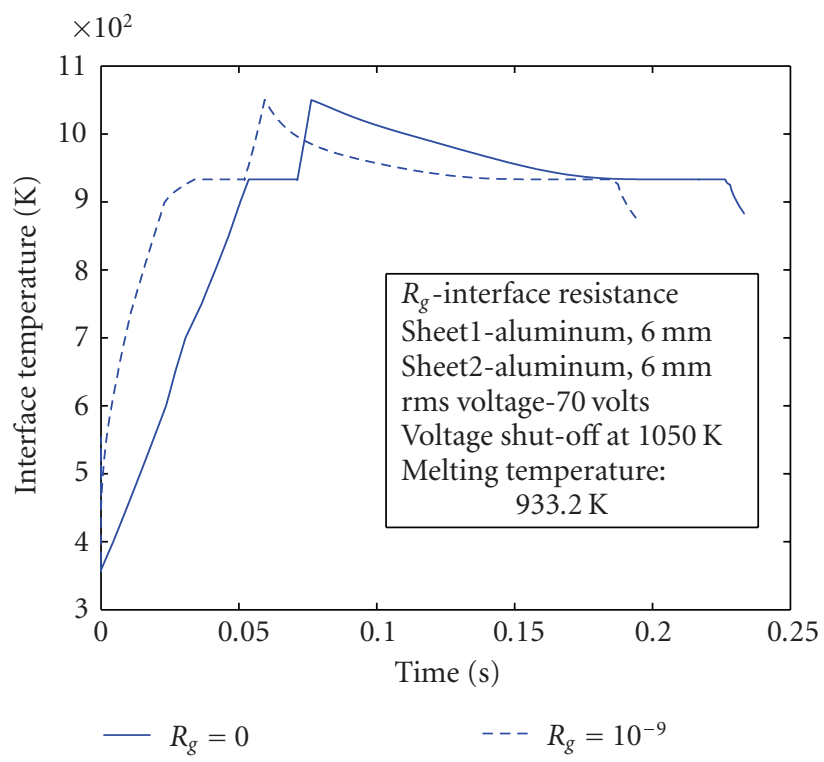

Figure 4.2. Example 1: temporal variation of interface temperature for two values of $R_{g}$.

As was mentioned above, a heat exchange condition might be more realistic. But, this requires experimental determination of the heat exchange coefficient, which we may do in future work.

4.1. Example 1: interface resistance. The first parameter examined is the process dependence on the interface resistance $R_{g}$. This resistance is meant to represent the effect of the microscopic gap between the two sheets of metal, as well as the effect of any coatings that may be applied by the user to improve the welding process. A number of tests were conducted, but only two representative cases are shown here. In both cases, two $6 \mathrm{~mm}$ aluminum plates were used.

In the first case, we set interface resistance $R_{g}=0$. As can be seen in Figure 4.2, the interface temperature in this case increases steadily with time until it reaches the melting temperature. The temperature then stays constant and is equal to the melting temperature for a short while before starting to increase again. The constant temperature period indicates the presence of a mixture of solid and liquid phases that characterizes the mushy region. As time increases, the fraction of liquid in the mixture increases until all of the solid has melted, at which point the temperature can increase again. In this case, the power was numerically shut off (by setting $V_{0}=0$ ) once the interface temperature reached a value of $1050 \mathrm{~K}$. The top two plots of Figure 4.3 show the spatial and temporal evolution of the temperature distribution through the plates during the melting and solidification phases. The lines show the temperature distribution for equal time intervals. 

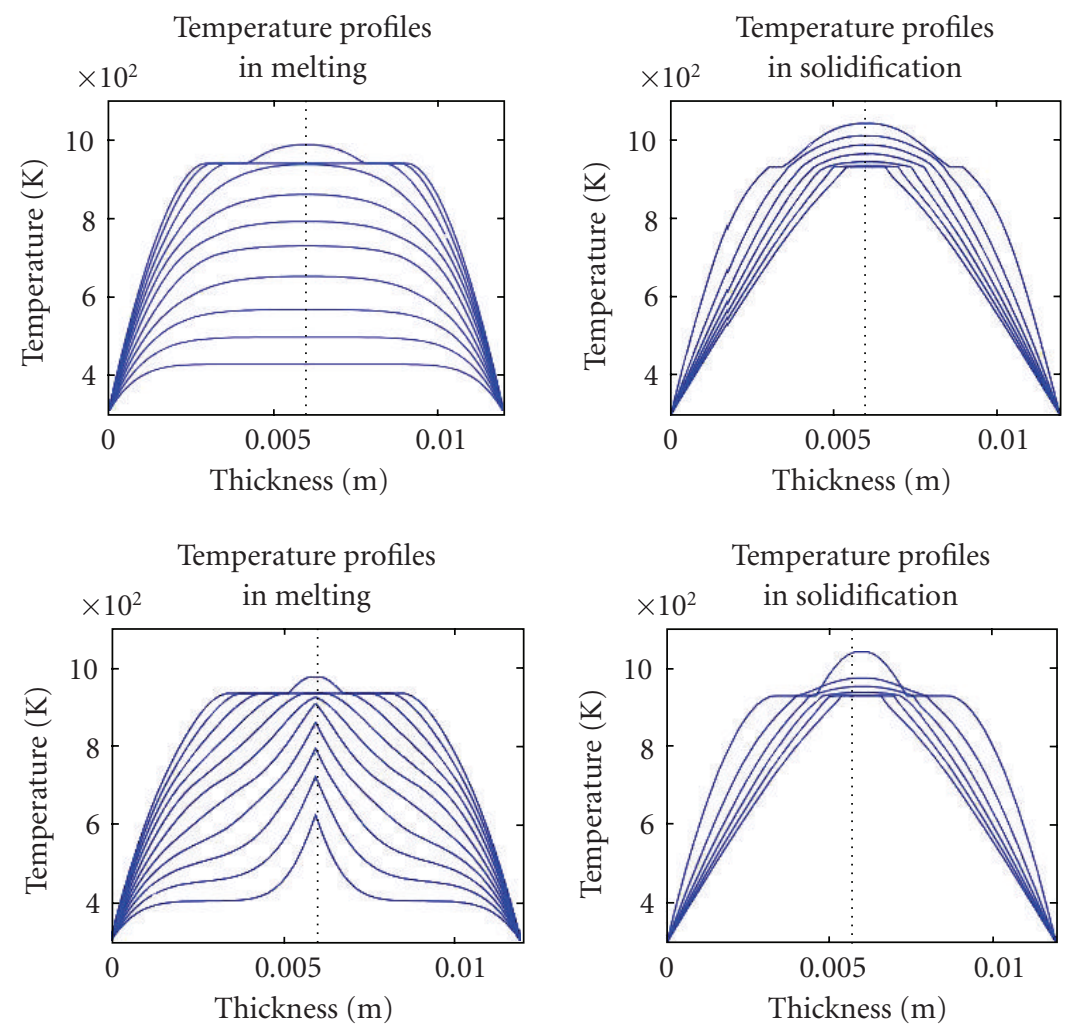

Figure 4.3. Example 1: spatial and temporal variation of temperature: $R_{g}=0$ (top), $R_{g}=10^{-9}$ initially (bottom). The curves represent equal time increments.

A number of observations can be made. First, the temperature profiles seem to be symmetrical as would be expected, and the peak temperature occurs at the interface between the two plates, that is, at $x=6 \mathrm{~mm}$. Due to a lack of a centralized "energy source," the temperature is almost uniform through the central portions of the plates. The development of mushy zones is apparent from the plateaus at $933.2 \mathrm{~K}$. The solidification plot shows the temperature dropping to the melting temperature and below once the voltage is turned off. The extent of the mushy and molten zones as a function of time can be seen in Figure 4.4. Melting is seen to first take place at the interface and then propagates into the plates as time increases. The darker symbols represent the maximum extent of the molten or liquid region $\left(\left\{\theta>\theta^{*}\right\}\right)$. The interface is the first to become fully liquid. The effect of voltage shut-off and solidification can be observed in the shrinking of the mushy and molten regions until no more liquid or mushy points are visible at later times, and the plates and the nugget are fully solid.

In the second case, we consider a resistance $R_{g}$ that is initially set equal to $10^{-9}$ and then decreases linearly to zero at the melting temperature. As can be seen from Figure 4.2, the interface temperature increases more rapidly than in the previous case and melting 

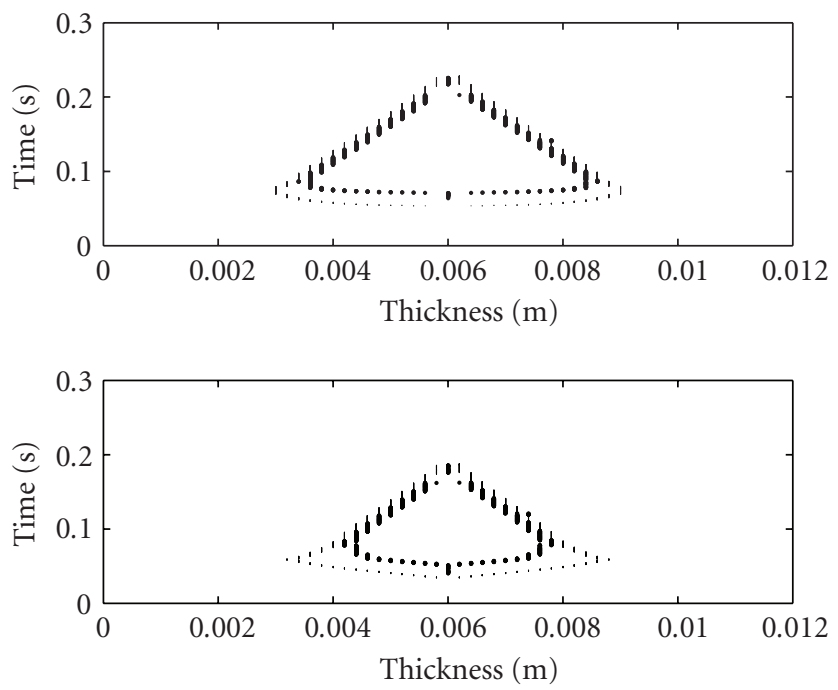

Figure 4.4. Example 1: extent of mushy and molten regions as a function of time for $R_{g}=0$ (top), $R_{g}=10^{-9}$ initially (bottom). Darker markers indicate molten zone; lighter markers indicate mushy zone.

occurs sooner. Also, the graphs are slightly different as the temperature approaches the melting point. The meeting of the graphs at time 0.005 second seems to be coincidental. Hence, it appears that the addition of an interface resistance results in faster melting. In Figure 4.3 , the temperature profile is seen to vary significantly from the $R_{g}=0$ case. The extra interface resistance results in localized heating and sharper temperature gradients at the interface. As in the previous case, a mushy zone can be observed. However, the maximum extent of the mushy and molten regions is lower by the time the voltage is shut off. In the case of $R_{g}=0$, the maximum extent of the mushy and molten regions, as measured from the interface, were $3 \mathrm{~mm}$ and $2.6 \mathrm{~mm}$, respectively. In the case of $R_{g}=$ $10^{-9}$, initially, these values dropped to $2.8 \mathrm{~mm}$ and $1.8 \mathrm{~mm}$, respectively. This molten region is what forms a weld nugget upon solidification. Thus increasing the interface resistance results in faster melting, but smaller nuggets, if the voltage shut-off point is set based on the interface temperature.

Figure 4.5 shows the spatial distribution of $F_{j}^{n}$, the discretized Joule heating source term $F$, through the two plates at a time instant prior to melting. It is clear that the Joule heating term is not uniformly distributed. The maximum Joule heating term occurs, as expected, at the location of the interface between the two plates. Indeed, according to (3.3), the Joule heating term is highest at the location with the lowest value of electrical conductivity, $\sigma$. As can be seen in Figure 4.1, the electrical resistivity increases with temperature, hence the electrical conductivity decreases with temperature. At any given time, the lowest electrical conductivity occurs at the location of maximum temperature, that is, the interface in this case. This clearly indicates that assuming some averaged uniform heat source is somewhat unrealistic. 


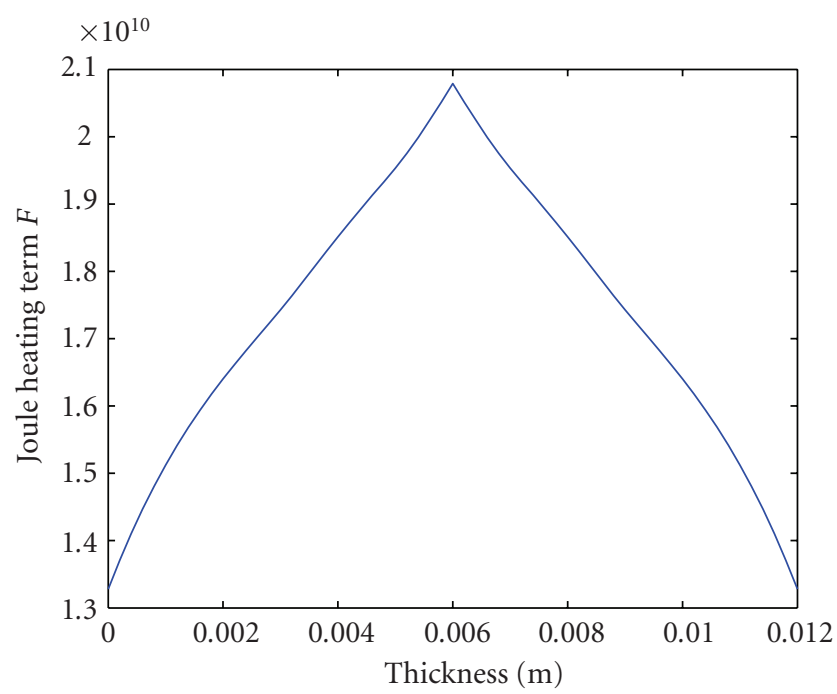

Figure 4.5. Example 1: the Joule heating source term as a function of $x$ at a fixed time for the case $R_{g}=10^{-9}$ initially.

For the sake of completeness, we depict in Figure 4.6 the development of the mushy and molten regions when there is no voltage shut-off. Then, as is to be expected $[4,12]$, the mushy region shrinks to a sharp interface, while the molten region reaches close to the outer boundary. As mentioned earlier, such growth of the molten region may lead to expulsion of molten metal, which causes considerable damage to the system. In the present formulation, expulsion does not occur due to the Dirichlet boundary condition used at the outer surfaces.

4.2. Example 2: plates of unequal thickness. The next test examined the effect of asymmetry on the temperature evolution. Here, the two aluminum plates had different thicknesses: plate 1 had a thickness $l=3 \mathrm{~mm}$ and plate 2 had a thickness $L-l=6 \mathrm{~mm}$. Two values of $R_{g}$ were again considered, again $R_{g}=0$ and $R_{g}=10^{-9}$ initially.

Figure 4.7 shows the spatial and temporal evolution of the temperature distribution through the plates during the melting and solidification phases. In the case of $R_{g}=0$, the temperature profile remains symmetrical and peak temperatures are observed at the midpoint, $x=4.5 \mathrm{~mm}$, instead of at the interface (indicated by the dashed line). This would be undesirable in a real application. As observed in the case of plates of equal thickness, the temperature profile is almost uniform over a significant portion of the two plates until melting starts to occur. However, when an initial resistance of $10^{-9}$ is introduced at the interface, peak temperatures and sharp gradients are once again first observed between the two plates at the interface, but eventually shift to the right (towards the center of the second plate). In fact, melting first occurs at a point slightly to the right of the interface. As time progresses, the peak temperature is seen shifting further away from the interface, and most of the melting occurs in the thicker plate. 


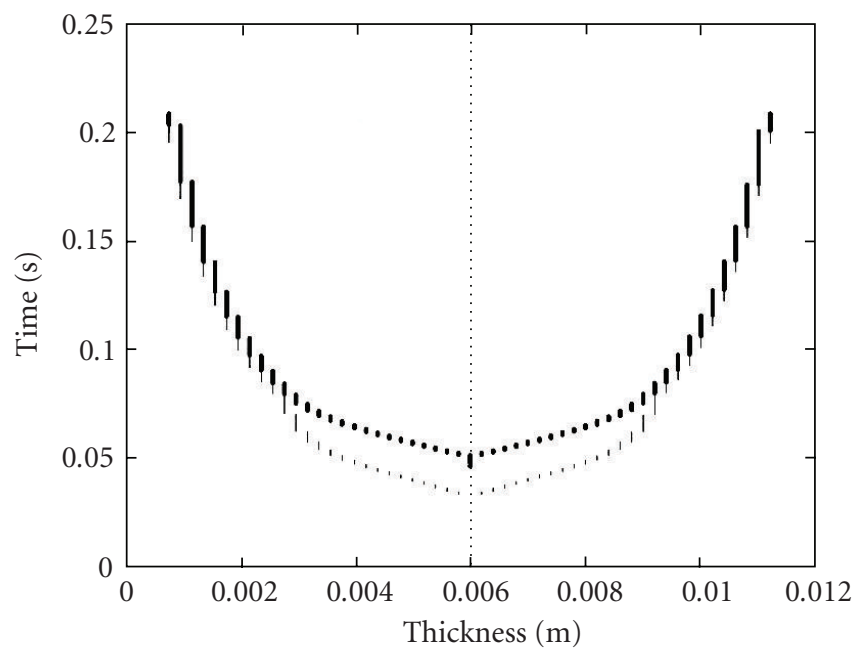

Figure 4.6. Example 1: extent of mushy and molten regions as a function of time for $R_{g}=10^{-9}$ initially without voltage shut-off. Darker markers indicate molten zone; lighter markers indicate mushy zone.

The development of the mushy and molten regions is shown in Figure 4.8. In the case of $R_{g}=0$, a mushy and molten material first appears at $x=4.5 \mathrm{~mm}$, the midpoint. Most of the nugget is located in the second plate and the liquid regions are symmetrical about the midpoint, not the interface. When a nonzero resistance $R_{g}$ is introduced at the interface, the melting shifts closer to the interface, and the nugget does not extend as deeply into the second plate. However, since $R_{g}$ is set to zero once the interface reaches the melting temperature, the two formulations (zero and nonzero initial $R_{g}$ values) become identical, and one would expect to see the peak temperature slowly shift towards the centerpoint, away from the interface.

Welding plates of different thickness seem to not be an easy task. These results indicate that the nugget location and size may be controlled to some extent by varying the interface resistance.

4.3. Example 3: comparison of DC and AC. The next test compared the effect of using a direct (DC) current with a $60 \mathrm{~Hz}$ alternating (AC) current on the welding process. Two aluminum plates, each with a thickness of $6 \mathrm{~mm}$, were considered. A linearly decreasing interface with an initial resistance value of $10^{-9}$ was assumed in both cases. In order to make the comparison more meaningful, the AC rms voltage was set equal to the DC constant voltage, that is, a constant voltage input of $50 \mathrm{~V}$ was used for the DC case, while an alternating voltage equal to $70.7 \sin (120 \pi t) \mathrm{V}$ was used in the $\mathrm{AC}$ case.

As can be seen in Figure 4.9, the interface temperature is seen to increase steadily in the DC case, while small oscillations can be seen in the AC case. The melting and voltage shut-off temperatures are reached sooner in the DC case. For the parameter values used, the preheat time (the time taken for the interface to reach the melting temperature) is 0.118 second for the DC case, versus 0.121 second for the AC case. The weld 

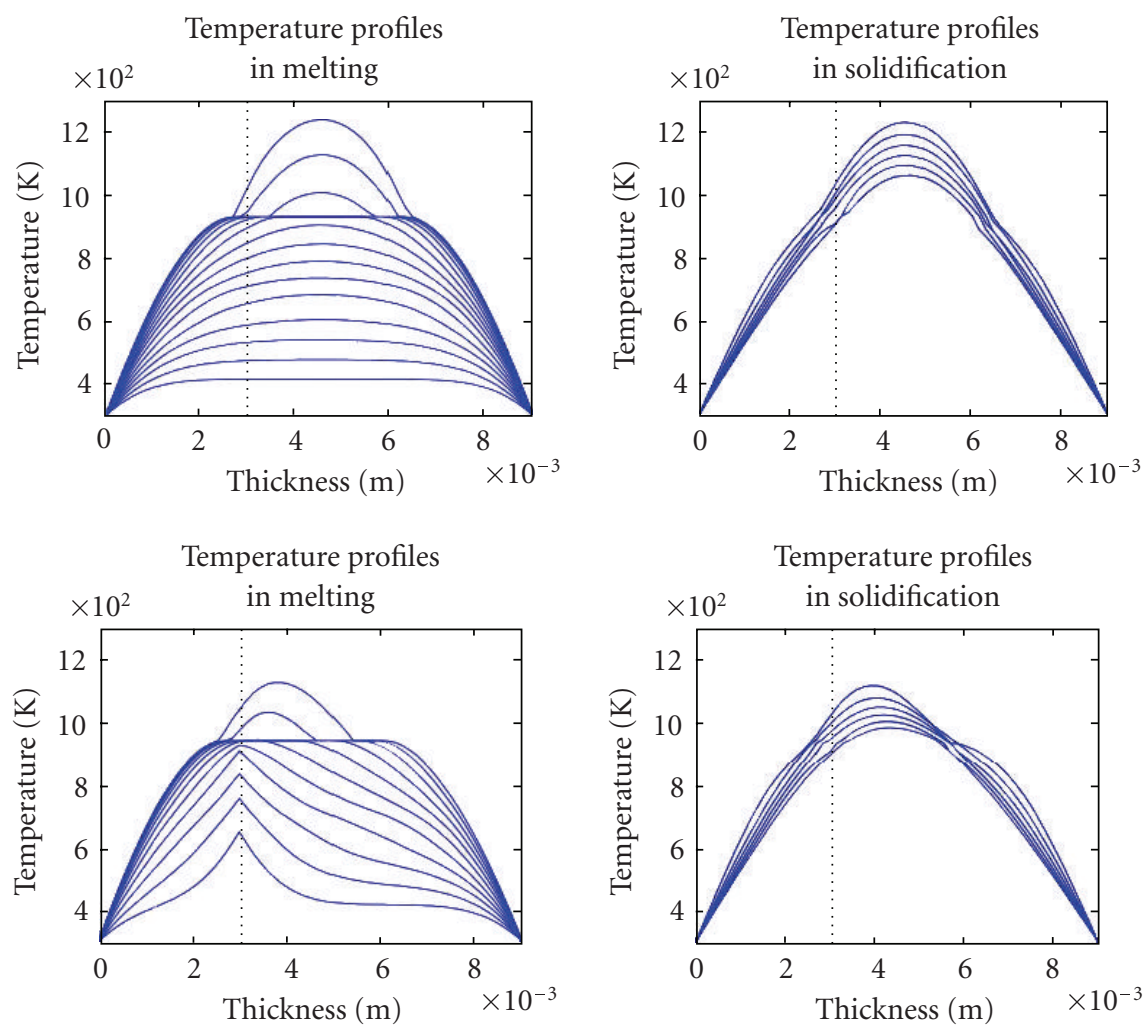

Figure 4.7. Example 2: spatial and temporal variation of temperature for $R_{g}=0$ (top), $R_{g}=10^{-9}$ initially (bottom). The curves represent equal time increments. Dotted line indicates location of interface.

time (the length of time that voltage or current is applied to the workpieces) drops from 0.171 second in the AC case to 0.165 second for the DC case. No further degradation of the $\mathrm{AC}$ input due to the power factor was considered here. However, these preliminary results indicate that a DC power input might be faster and more effective than an AC power input. Clearly, a more thorough investigation is needed, since the differences observed in this example are rather small. We note that the small difference may be also attributed to the fact that the time scale associated with the AC current input, on the order of 0.02 second, is much smaller than the melting time scale, which is on the order of 0.1 second.

This model is clearly a tool which may be used to investigate more thoroughly the possible differences in the use of AC or DC currents in spot welding.

4.4. Welding of dissimilar materials. The final simulations deal with the welding of plates made of dissimilar materials. The plates were assumed to have equal thickness of $6 \mathrm{~mm}$. The left plate was made of steel with melting temperature of $1809 \mathrm{~K}$ and electrical 


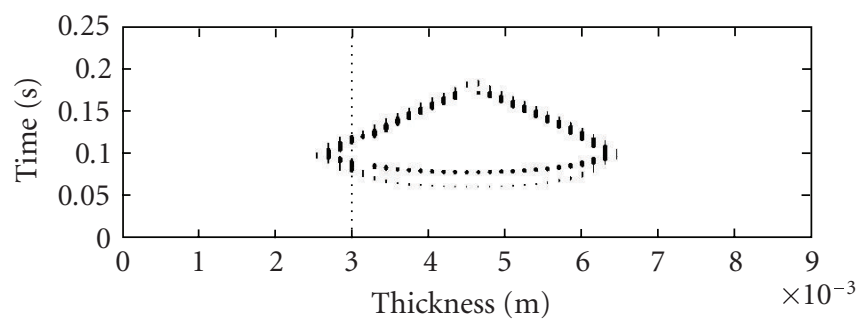

(a)

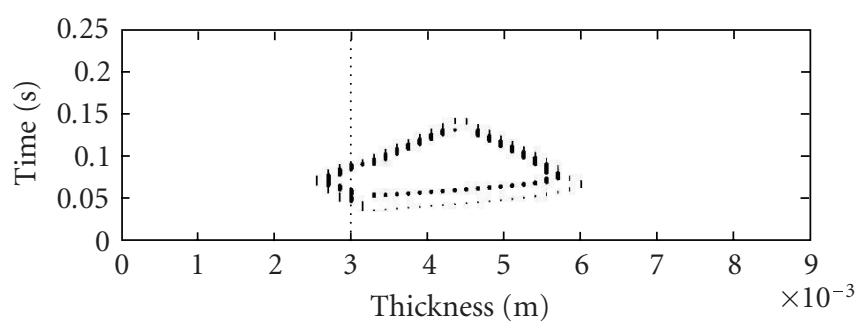

(b)

Figure 4.8. Example 2: extent of mushy and molten regions as a function of time for $R_{g}=0$ (a), $R_{g}=10^{-9}$ initially (b). Darker markers indicate molten zone; lighter markers indicate mushy zone. Dotted line indicates location of interface.

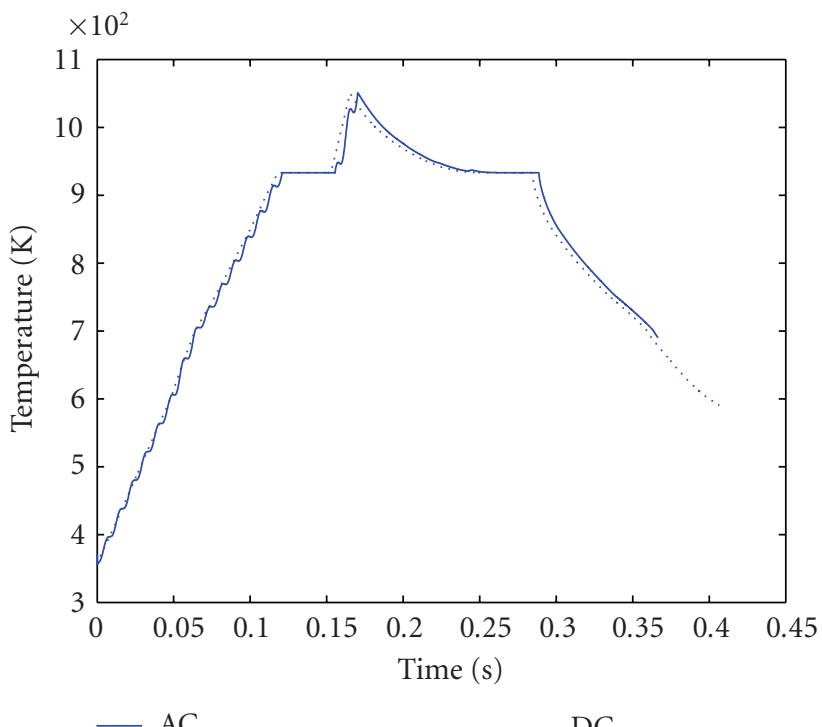

Figure 4.9. Example 3: temporal variation of interface temperature for $\mathrm{AC}$ and DC current input. 


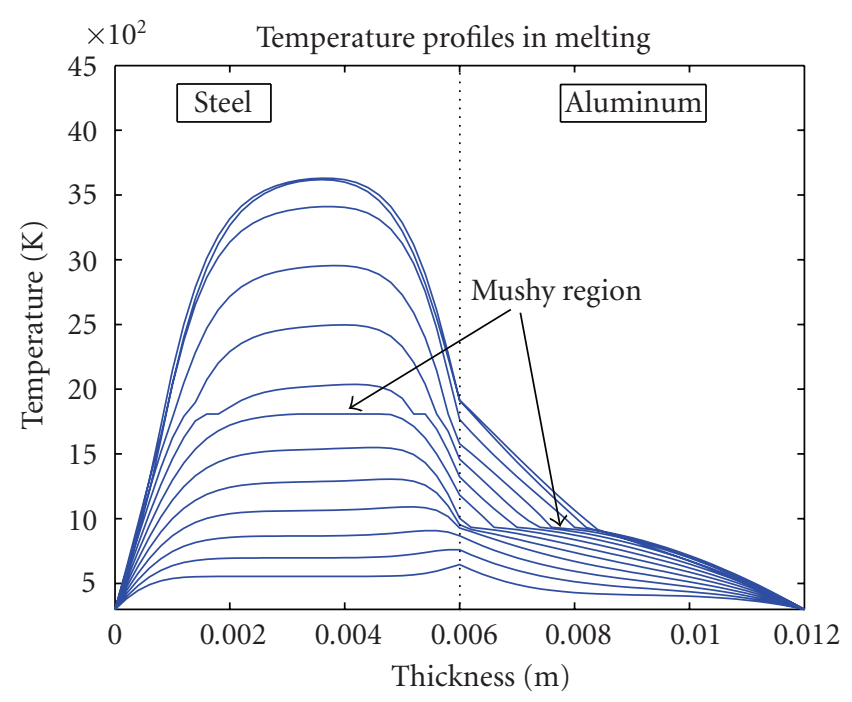

Figure 4.10. Example 4: spatial and temporal variation of temperature. The dotted line is the interface between the plates. The curves represent equal time increments.

resistivity of $1.225 \times 10^{-7} \Omega \cdot \mathrm{m}$ at $300 \mathrm{~K}$, while the right plate was made of aluminum with electrical resistivity of $5.42 \times 10^{-6} \Omega \cdot \mathrm{m}$ at $300 \mathrm{~K}$. The DC voltage was shut off when the interface temperature reached $1900 \mathrm{~K}$.

The results of the simulations are depicted in Figure 4.10, where the curves of temperature versus $x$ are plotted for different times with equal increments.

Compared to the earlier examples, the behavior of the system is quite different. Aluminum starts melting first, and a mushy region develops in that plate. The steel plate starts melting later, and also develops a mushy region. The important features seen in the figure are the steep temperature gradient in the molten aluminum, as well as the extent of the mushy and molten regions in each plate. Since the extent of the molten region is substantial, as compared to the plate thickness, there may be mixing of the liquid metals, a fact that is likely to have some influence on the nugget composition and strength, as well as on the liquid metal properties. Moreover, if the boundary condition is changed to heat exchange form, it is very likely that expulsion of the molten material will occur.

The asymmetry and the extent of the molten and mushy regions in the steel and the aluminum are clearly depicted in Figure 4.11. Moreover, it is seen that the melting of the steel starts in the interior of the steel plate and reaches the other plate only some time later, while the aluminum starts melting at the interface.

\section{Conclusions}

This work constructs a sophisticated one-dimensional model for the spot-welding process. It is based on the enthalpy approach and takes into account the temperature dependence of the various process parameters, and, in particular, their variation in the mushy region. It also takes into account the resistance of the outer electrical circuit, the 


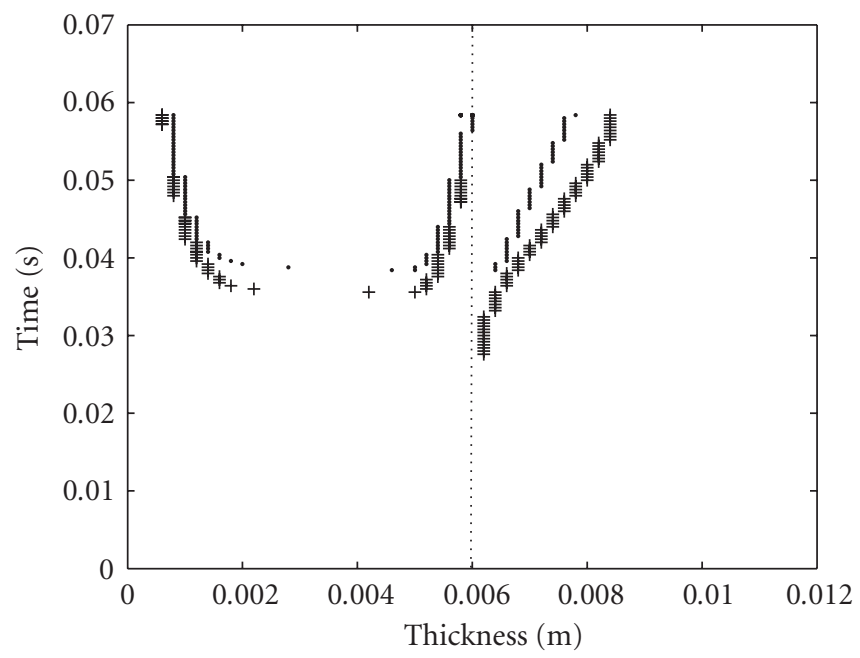

Figure 4.11. Example 4: the mushy (+ signs) and molten (other signs) regions in the steel (left of the broken line) and the aluminum (right of the broken line) plates.

electrodes, and the surface resistance between the plates. The latter can be attributed to the imperfect thermal and electrical contacts between the solid plates and to the possible addition of a thin layer of a higher resistance material between the plates. This addition is done to enhance and better control the welding outcome. These features are rarely found in the usual models and computer software for spot welding.

This model is meant as a tool for the design engineer to obtain quickly a reasonable estimate of the performance of the welding process under design, as well as a good indication of an efficient welding schedule.

A numerical algorithm, based on finite differences, is presented and the results of computer simulations obtained from a code, based on the algorithm, are depicted. It is clear from the simulations that the model and the algorithm are very useful tools in the study of the process dependence on the setting and the parameters. In this work, because of lack of experimental data, we used simple interpolation of the parameters, especially in the mushy region. However, in view of Figure 4.1, this maybe somewhat simplistic.

We performed a large number of numerical simulations. Here we report on four of them. In Example 1, we see that increasing the interface resistance results in faster melting but smaller nuggets. Example 2 shows that welding of plates of different thickness present special difficulties in nugget location and size which may be partially controlled by interface resistance. In the setting of Example 3, there seems to be very little difference between using AC or DC currents in the spot welding process. Finally, Example 4 illustrates the difficulties that one may encounter when welding materials with very different melting temperatures and properties.

In the construction of the model, we have encountered the usual problem that some of the desired parameter values could not be found in the literature. In particular, there is essentially no information about the variation of the process parameters such as specific 
heat or electric resistance in the mushy region. Such information needs to be obtained if accurate process control is to be achieved, and there is also a need to experimentally and theoretically study in detail the evolution and structure of mushy regions. Furthermore, work needs to be done to reasonably quantify the values of temperature dependence of the interface resistance $R_{g}$.

Because the model is one-dimensional, it is likely that the description of the electric current flow is rather inaccurate, as compared to a three-dimensional formulation. However, this simplification may introduce only a small distortion. This issue will be studied in the following stages of this work where we will extend the model and the numerical algorithm to two and three dimensions.

Although the literature on spot welding is extensive, this work clearly indicates that much still needs to be done. In addition to the mathematical analysis of the model, and proofs of the convergence of the numerical scheme, the following issues need to be addressed.

(i) The dependence of the parameters on temperature, and their values in the mushy region are needed.

(ii) The contact resistance between the plates depends also on the contact pressure that the electrodes exert on the plates, which was not taken into account in the model. This contact pressure may cause deformation of the plates and the heating of the electrodes may cause distortions.

(iii) A heat exchange condition between the electrodes and the plates may be a more realistic description of the process. The use of this condition may show that under certain operating conditions expulsion of the molten metal may occur.

(iv) Quantification of the contribution of surface coatings to the interface resistance $R_{g}$ is needed.

\section{Acknowledgment}

The authors would like to thank the two referees for their constructive comments which have improved the manuscript.

\section{References}

[1] “Technology for efficient and accurate spot welding," Technology and Products Section, JETRO, November 1995, 24-25.

[2] D. J. Browne, H. W. Chandler, J. T. Evans, and I. Wen, "Computer simulation of resistance spot welding in aluminum—part 1," Welding Journal, vol. 74, no. 10, pp. 339-344, 1995.

[3] J. Crank, Free and Moving Boundary Problems, Oxford Science Publications, The Clarendon Press, Oxford University Press, New York, 1984.

[4] C. M. Elliott and J. R. Ockendon, Weak and Variational Methods for Moving Boundary Problems, vol. 59 of Research Notes in Mathematics, Pitman, Massachusetts, 1982.

[5] E. Feulvarch, V. Robin, and J. M. Bergheau, "Resistance spot welding simulation: a general finite element formulation of electro thermal contact conditions," Journal of Materials Processing Technology, vol. 153-154, pp. 436-441, 2004.

[6] R. M. Furzeland, "A comparative study of numerical methods for moving boundary problems," Journal of the Institute of Mathematics and Its Applications, vol. 26, no. 4, pp. 411-429, 1980.

[7] R. F. Gariepy, M. Shillor, and X. Xu, "Existence of generalized weak solutions to a model for in situ vitrification," European Journal of Applied Mathematics, vol. 9, no. 6, pp. 543-559, 1998. 
[8] J. E. Gould, "An examination of Nugget development during spot welding using both experimental and analytical techniques," Welding Journal, vol. 66, no. 1, pp. 1-10, 1987.

[9] S. D. Howison, J. F. Rodrigues, and M. Shillor, "Stationary solutions to the thermistor problem," Journal of Mathematical Analysis and Applications, vol. 174, no. 2, pp. 573-588, 1993.

[10] J. A. Khan, K. Broach, and A. Kabir, "Numerical thermal model of resistance spot welding in aluminum," Journal of Thermophysics and Heat Transfer, vol. 14, no. 1, pp. 88-95, 2000.

[11] J. A. Khan, L. Xu, and Y.-J. Chao, "Prediction of nugget development during resistance spot welding using coupled thermal-electrical-mechanical model," Science and Technology of Welding and Joining, vol. 4, no. 4, pp. 201-207, 1999.

[12] A. A. Lacey and M. Shillor, "The existence and stability of regions with superheating in the classical two-phase one-dimensional Stefan problem with heat sources," IMA Journal of Applied Mathematics, vol. 30, no. 2, pp. 215-230, 1983.

[13] A. A. Lacey and A. B. Tayler, "A mushy region in a Stefan problem," IMA Journal of Applied Mathematics, vol. 30, no. 3, pp. 303-313, 1983.

[14] J. A. Mackenzie and M. L. Robertson, "The numerical solution of one-dimensional phase change problems using an adaptive moving mesh method," Journal of Computational Physics, vol. 161, no. 2, pp. 537-557, 2000.

[15] P. Shi, M. Shillor, and X. Xu, "Existence of a solution to the Stefan problem with Joule's heating," Journal of Differential Equations, vol. 105, no. 2, pp. 239-263, 1993.

[16] X. Sun and M. A. Khaleel, "Resistance spot welding of aluminum alloy to steel with transition material—part 2: finite element analysis of nugget growth," Welding Journal, vol. 83, no. 7, pp. 197-202, 2004.

[17] A. B. Tayler, Mathematical Models in Applied Mechanics, Oxford Applied Mathematics and Computing Science Series, The Clarendon Press, Oxford University Press, New York, 1986.

[18] L. Wei, D. Cerjanec, and G. A. Grzadzinski, "A comparative study of single-phase AC and multiphase DC resistance spot welding," Journal of Manufacturing Science and Engineering, vol. 127, no. 8, pp. 583-589, 2005.

[19] X. Xu and M. Shillor, "The Stefan problem with convection and Joule's heating," Advances in Differential Equations, vol. 2, no. 4, pp. 667-691, 1997.

[20] L. S. Yao and J. Prusa, "Melting and freezing," in Advances in Heat transfer, vol. 19, pp. 1-95, 1989.

K. T. Andrews: Department of Mathematics and Statistics, Oakland University, Rochester, MI 48309-4478, USA

E-mail address: andrews@oakland.edu

L. Guessous: Department of Mechanical Engineering, Oakland University, Rochester,

MI 48309-4478, USA

E-mail address: guessous@oakland.edu

S. Nassar: Department of Mechanical Engineering, Oakland University, Rochester,

MI 48309-4478, USA

E-mail address: nassar@oakland.edu

S. V. Putta: Department of Mechanical Engineering, Oakland University, Rochester, MI 48309-4478, USA

E-mail address: svputta@oakland.edu

M. Shillor: Department of Mathematics and Statistics, Oakland University, Rochester,

MI 48309-4478, USA

E-mail address: shillor@oakland.edu 


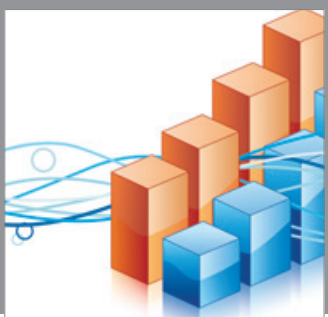

Advances in

Operations Research

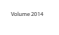

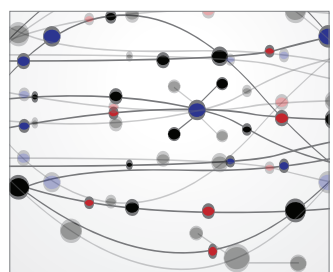

\section{The Scientific} World Journal
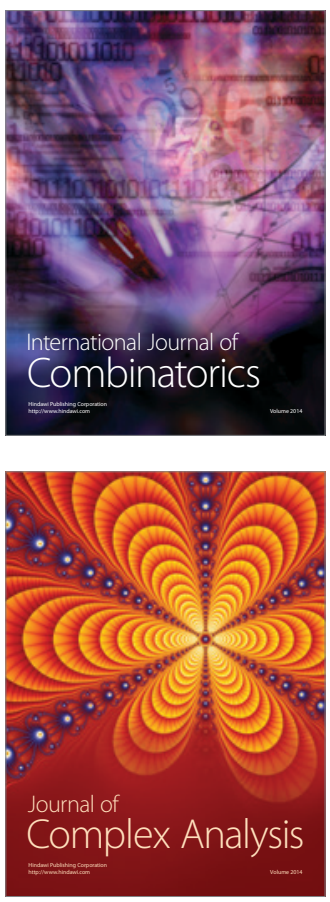

International Journal of

Mathematics and

Mathematical

Sciences
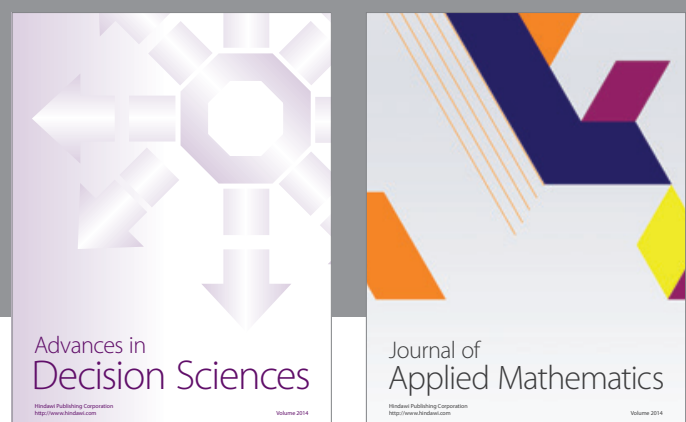

Journal of

Applied Mathematics
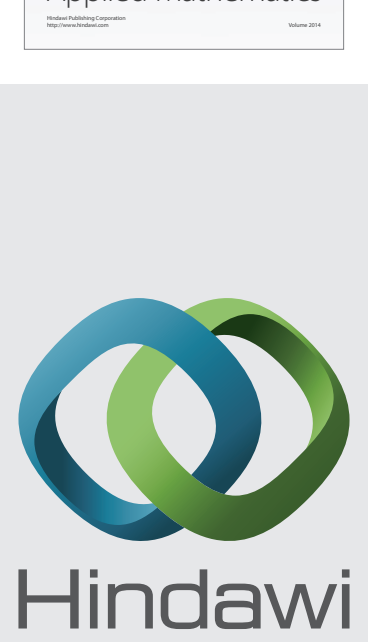

Submit your manuscripts at http://www.hindawi.com
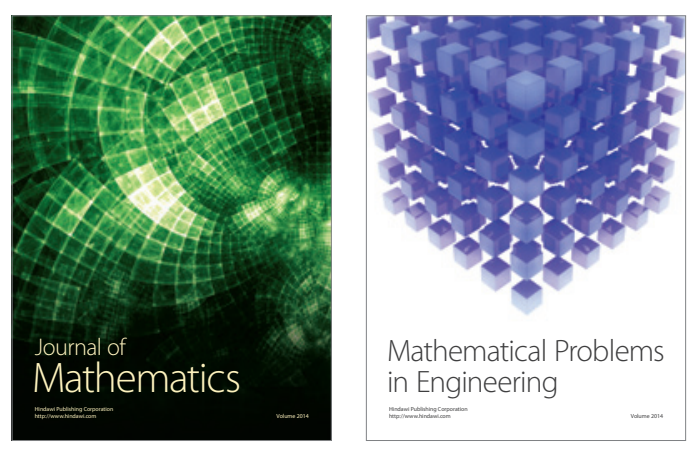

Mathematical Problems in Engineering
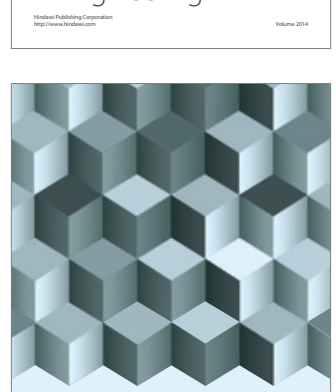

Journal of

Function Spaces
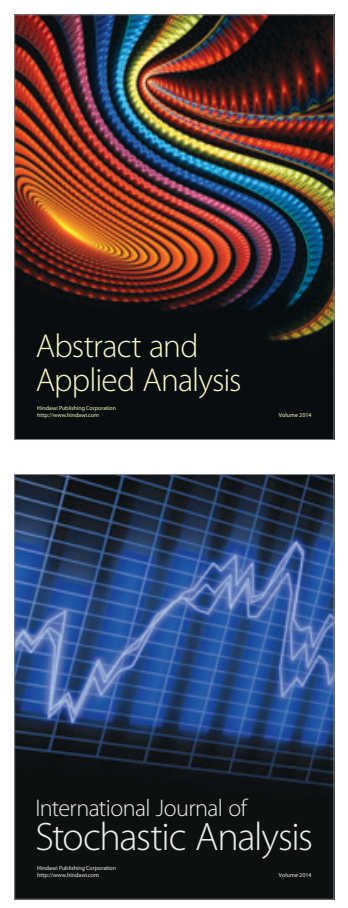

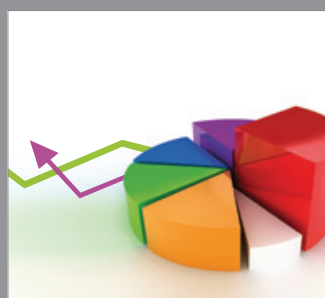

ournal of

Probability and Statistics

Promensencen
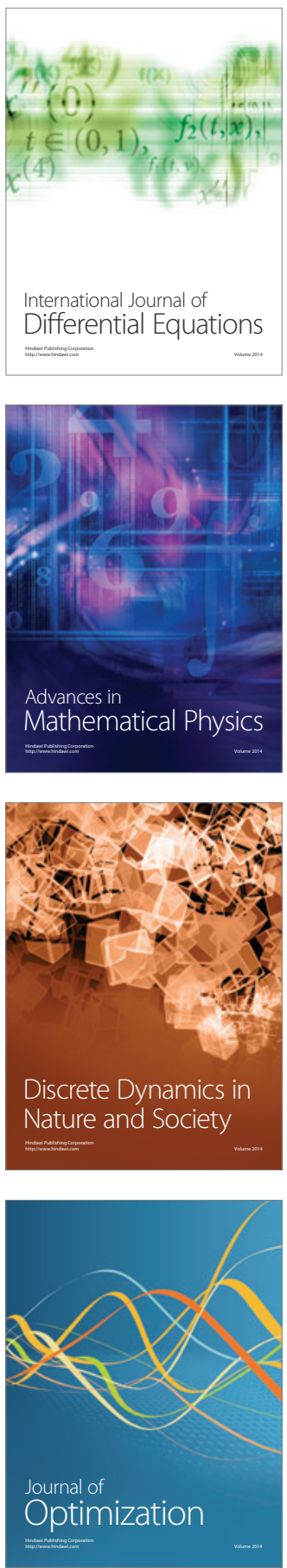